\title{
Electricity savings and greenhouse gas emission reductions from global phase-down of hydrofluorocarbons
}

Pallav Purohit ${ }^{1}$, Lena Höglund-Isaksson ${ }^{1}$, John Dulac ${ }^{2}$, Nihar Shah ${ }^{3}$, Max Wei ${ }^{3}$, Peter Rafaj ${ }^{1}$, and Wolfgang Schöpp ${ }^{1}$

${ }^{1}$ Air Quality and Greenhouse Gases (AIR) Program, International Institute for Applied Systems Analysis (IIASA), Schlossplatz 1, A-2361, Laxenburg, Austria

${ }^{2}$ International Energy Agency (IEA), 9, rue de la Federation, 75015 Paris, France

${ }^{3}$ Energy Technology Area, Lawrence Berkeley National Laboratory (LBNL), 1 Cyclotron Road, Berkeley, CA 94720, USA

Correspondence to: Pallav Purohit (purohit@iiiasa.ac.at) 


\section{S1: Current legislation on HFC control in the Baselines}

To estimate hydrofluorocarbon (HFC) emissions in the baseline scenarios, we take into account the effects on emissions from implementation of existing legislations to control HFC emissions at the regional or national level. The European Union (EU) first legislated to control emissions of HFCs in 2006 (Höglund-Isaksson et al., 2012; Höglund-Isaksson et al., 2013), adopting a regulation on emissions and a directive on mobile air-conditioning (EU, 2006). Regulation 842/2006 on certain fluorinated greenhouse gases (GHGs) aimed only at containment, through measures such as control of leaks, proper servicing of equipment and recovery of the gases at the end of the equipment's life. In May 2014, this was replaced by the much more ambitious Regulation 517/2014 on fluorinated GHGs (the F-Gas Regulation ${ }^{1}$ ), which entered into force on $1^{\text {st }}$ January 2015. It is aimed at achieving a reduction in sales of HFCs on the EU market by 79 percent (GWP-weighted) from 2009-12 levels by 2030 (EU, 2014), with interim reduction steps starting in 2015 and applying roughly every three years. In addition, HFCs are banned outright in some categories of new equipment where alternatives are readily available.

Apart from the EU, Japan, the USA, Australia, Norway, and Switzerland have also implemented national regulations to limit the use of high-GWP HFCs. The USA has already implemented incentive credits for use of low-GWP refrigerants in support of greenhouse gas emission standards for light duty vehicles and removed certain high-GWP HFCs from the Significant New Alternatives Policy (SNAP) list of allowable technologies for specific sectors in 2015 (US EPA, 2015). Although recently legally challenged (Reilly, 2017), the changes to the SNAP list ban the use of many high-GWP HFCs in, for example, commercial refrigeration applications, such as supermarket systems and vending machines, beginning during the period 20162020 and in mobile air-conditioning from model year 2021. The implementation of the new SNAP list is estimated to reduce baseline USA HFC emissions by $0.18-0.24$ Gt $\mathrm{CO}_{2}$ eq per year (about 43\%) by 2050 (Velders et al., 2015). In Japan, the Fluorocarbons Recovery and Destruction Law was amended and became effective on $1^{\text {st }}$ April 2015 as the Act on Rational Use and Proper Management of Fluorocarbons (Fluorocarbon Emission Control Law) (METI, 2015). Among other requirements, the Act requires entities manufacturing and importing air-conditioning and refrigeration units to transition to either fluorocarbon-free refrigerants or to low global warming fluorocarbons by certain target years.

For developing countries, several studies discuss the impact of the Clean Development Mechanism (CDM) projects on HFC23 emissions from HCFC-22 production for emissive and feedstock applications (Wara, 2007; Miller et al., 2010; Montzka et al., 2010; Miller and Kuijpers, 2011; Schneider, 2011). HFC-23 emissions from HCFC-22 production are assumed to be controlled in most developing countries due to CDM (Fenhann, 2014), except China where 36\% of HCFC-22 production is controlled (Feng et al., 2012). The Chinese production capacity of HCFC-22 accounts for 78\% of the global HCFC production (UNEP, 2014). HCFC-22 is a major source of HFC-23 emissions, which is a strong greenhouse gas with GWP 100 of 14,800 times that of $\mathrm{CO}_{2}$ (IPCC, 2007). 
In its Intended Nationally Determined Contribution (INDC) submitted in June 2015, China reiterated its commitment under the Montreal Protocol to achieve effective control on emissions of HFC-23 by 2020. In 2015, the Chinese National Development and Reform Commission (NDRC) announced that it plans to achieve abatement of all HFC-23 emissions by 2019 (NDRC, 2015). This would imply installing destruction technology in all plants currently not covered by CDM and ensuring that the destruction technology on plants covered under CDM is being operated and maintained. In line with this information, we assume in recent updates of the GAINS model that all HCFC-22 production facilities in China will be fully controlled from 2020 onwards. It is observed that except for China other developing countries do not make HFC specific emission reduction commitments in the INDCs. In the $28^{\text {th }}$ Meeting of the Parties to the Montreal Protocol in October 2016 in Kigali, the Indian government presented a domestic legislation that mandates control of trifluoromethane (HFC-23) emissions. At present, all HCFC-22 production facilities in India are fully controlled under the Clean Development Mechanism (CDM) and we assume the control on all Indian facilities stays operational and will be maintained in the future.

\section{S2: The Kigali Amendment to the Montreal Protocol}

The Kigali Amendment to the Montreal Protocol on Substances that Deplete the Ozone Layer entered into force on $1^{\text {st }}$ January 2019 , following ratification by 65 countries ${ }^{1}$. KA sets targets for the phase-down of HFCs consumption for four different Party groups. The first group primarily includes 136 developing countries that make up all Article 5 countries as specified under the Montreal Protocol with the exception of Bahrain, India, Iran, Iraq, Kuwait, Oman, Pakistan, Qatar, Saudi Arabia, and the United Arab Emirates (UAE). These ten countries are characterized by high ambient air temperatures and make up a second and separate group of Article 5 countries. Countries specified as non-Article 5 countries under the Montreal Protocol are primarily developed countries and under the Kigali Amendment divided into two separate groups with 45 countries in a first group and with the five countries Belarus, the Russian Federation, Kazakhstan, Tajikistan and Uzbekistan forming a separate second group. Table 1 presents the baseline years and HFC phase-down schedule of Article 5 and non-Article 5 Parties. We will hereafter refer to these four Party groups as Article 5 Group I, Article 5 Group II, non-Article 5 Group I, and non-Article 5 Group II.

\footnotetext{
${ }^{1}$ The Parties to the Montreal Protocol agreed that the Kigali amendment to the Montreal Protocol would enter into force on 1 January 2019, provided that at least 20 Parties had ratified it.
} 
Table S1: Baseline and HFC phase-down schedule of Article-5 and non- Article-5 Parties

\begin{tabular}{|c|c|c|c|c|}
\hline & \multicolumn{2}{|c|}{ Article 5 (A5) Parties: Group I } & \multicolumn{2}{|c|}{ Article 5 (A5) Parties: Group II } \\
\hline Baseline Years & \multicolumn{2}{|c|}{$2020,2021 \& 2022$} & \multicolumn{2}{|c|}{$2024,2025 \& 2026$} \\
\hline $\begin{array}{l}\text { Baseline } \\
\text { calculation }\end{array}$ & \multicolumn{2}{|c|}{$\begin{array}{l}\text { Average production /consumption of } \\
\text { HFCs in 2020, 2021, and } 2022 \\
\text { plus } 65 \% \text { of HCFC baseline } \\
\text { production/consumption }\end{array}$} & \multicolumn{2}{|c|}{$\begin{array}{l}\text { Average production /consumption of } \\
\text { HFCs in } 2024,2025 \text {, and } 2026 \\
\text { plus } 65 \% \text { of HCFC baseline } \\
\text { production/consumption }\end{array}$} \\
\hline $\begin{array}{l}\text { Reduction steps } \\
\text { Freeze }\end{array}$ & \multicolumn{2}{|c|}{2024} & \multicolumn{2}{|c|}{2028} \\
\hline Step 1 & 2029 & $10 \%$ & 2032 & $10 \%$ \\
\hline Step 2 & 2035 & $30 \%$ & 2037 & $20 \%$ \\
\hline Step 3 & 2040 & $50 \%$ & 2042 & $30 \%$ \\
\hline \multirow[t]{2}{*}{ Step 4} & 2045 & $80 \%$ & 2047 & $85 \%$ \\
\hline & \multicolumn{2}{|c|}{ Non-Article 5 (non-A5): Group I } & \multicolumn{2}{|c|}{ Non-Article 5 (non-A5): Group II } \\
\hline Baseline Years & \multicolumn{2}{|c|}{$2011,2012 \& 2013$} & \multicolumn{2}{|c|}{$2011,2012 \& 2013$} \\
\hline $\begin{array}{l}\text { Baseline } \\
\text { Calculation }\end{array}$ & \multicolumn{2}{|c|}{$\begin{array}{l}\text { Average production /consumption of } \\
\text { HFCs in } 2011,2012 \& 2013 \\
\text { plus } \mathbf{1 5 \%} \text { of HCFC baseline } \\
\text { production/consumption }\end{array}$} & \multicolumn{2}{|c|}{$\begin{array}{l}\text { Average production /consumption of } \\
\text { HFCs in } 2011,2012 \& 2013 \\
\text { plus } \mathbf{2 5 \%} \text { of HCFC baseline } \\
\text { production/consumption }\end{array}$} \\
\hline $\begin{array}{l}\text { Reduction steps } \\
\text { Step } 1\end{array}$ & 2019 & $10 \%$ & 2020 & $5 \%$ \\
\hline Step 2 & 2024 & $40 \%$ & 2025 & $35 \%$ \\
\hline Step 3 & 2029 & $70 \%$ & 2029 & $70 \%$ \\
\hline Step 4 & 2034 & $80 \%$ & 2034 & $80 \%$ \\
\hline Step 5 & 2036 & $85 \%$ & 2036 & $85 \%$ \\
\hline
\end{tabular}

Source: UNEP (2016) 
Table S2: Technical and economic energy efficiency (EE) potential of cooling technologies in baseline and with low-GWP alternatives

\begin{tabular}{|c|c|c|c|c|c|c|c|}
\hline \multirow[t]{3}{*}{ Countries } & \multirow[t]{3}{*}{ Year } & \multicolumn{6}{|c|}{ EE due to systems improvements and low-GWP refrigerant (\%) } \\
\hline & & \multicolumn{2}{|c|}{ HCFC-22/HFC-410A } & \multicolumn{2}{|c|}{ HC-290 } & \multicolumn{2}{|c|}{ HFC-32/ HFOs } \\
\hline & & Economic & Technical & Economic & Technical & Economic & Technical \\
\hline \multicolumn{8}{|c|}{ 1. Mini-split air-conditioners (1.5 ton base-unit size) } \\
\hline Brazil & 2014 & $30 \%$ & $70 \%$ & $36 \%$ & $73 \%$ & $35 \%$ & $72 \%$ \\
\hline Chile & 2014 & $30 \%$ & $70 \%$ & $36 \%$ & $73 \%$ & $35 \%$ & $72 \%$ \\
\hline China & 2014 & $30 \%$ & $70 \%$ & $36 \%$ & $73 \%$ & $35 \%$ & $72 \%$ \\
\hline Colombia & 2014 & $30 \%$ & $70 \%$ & $36 \%$ & $73 \%$ & $35 \%$ & $72 \%$ \\
\hline Egypt & 2014 & $30 \%$ & $70 \%$ & $36 \%$ & $73 \%$ & $35 \%$ & $72 \%$ \\
\hline India & 2014 & $30 \%$ & $70 \%$ & $36 \%$ & $73 \%$ & $35 \%$ & $72 \%$ \\
\hline Indonesia & 2014 & $30 \%$ & $70 \%$ & $36 \%$ & $73 \%$ & $35 \%$ & $72 \%$ \\
\hline Mexico & 2014 & $30 \%$ & $70 \%$ & $36 \%$ & $73 \%$ & $35 \%$ & $72 \%$ \\
\hline Pakistan & 2014 & $30 \%$ & $70 \%$ & $36 \%$ & $73 \%$ & $35 \%$ & $72 \%$ \\
\hline Saudi Arabia & 2014 & $30 \%$ & $70 \%$ & $36 \%$ & $73 \%$ & $35 \%$ & $72 \%$ \\
\hline Thailand & 2014 & $30 \%$ & $70 \%$ & $36 \%$ & $73 \%$ & $35 \%$ & $72 \%$ \\
\hline UAE & 2014 & $30 \%$ & $70 \%$ & $36 \%$ & $73 \%$ & $35 \%$ & $72 \%$ \\
\hline Vietnam & 2014 & $30 \%$ & $70 \%$ & $36 \%$ & $73 \%$ & $35 \%$ & $72 \%$ \\
\hline \multicolumn{8}{|c|}{ 2. Packaged air-conditioners (Rooftop units 10 ton AC) } \\
\hline Asia & 2015 & $31 \%$ & $49 \%$ & & & $37 \%$ & $53 \%$ \\
\hline North America & 2015 & $31 \%$ & $49 \%$ & & & $37 \%$ & $53 \%$ \\
\hline Europe & 2015 & $31 \%$ & $49 \%$ & & & $37 \%$ & $53 \%$ \\
\hline Rest of World & 2015 & $31 \%$ & $49 \%$ & & & $37 \%$ & $53 \%$ \\
\hline \multicolumn{8}{|c|}{ 3. VRF/ Ducted air-conditioners (10 ton HP) } \\
\hline Asia & 2015 & $15 \%$ & $37 \%$ & & & $21 \%$ & $41 \%$ \\
\hline North America & 2015 & $15 \%$ & $37 \%$ & & & $21 \%$ & $41 \%$ \\
\hline Europe & 2015 & $15 \%$ & $37 \%$ & & & $21 \%$ & $41 \%$ \\
\hline Rest of World & 2015 & $15 \%$ & $37 \%$ & & & $21 \%$ & $41 \%$ \\
\hline \multicolumn{8}{|c|}{ 4. Chillers, air cooled (Small, $<300$ tons $-500 \mathrm{~kW}, 143$ tons) ${ }^{+}$} \\
\hline Asia & $\sim 2012-2017$ & $29 \%$ & $38 \%$ & & & $32 \%$ & $41 \%$ \\
\hline North America & $\sim 2012-2017$ & $22 \%$ & $32 \%$ & & & $25 \%$ & $35 \%$ \\
\hline Europe & $\sim 2012-2017$ & $33 \%$ & $42 \%$ & & & $36 \%$ & $44 \%$ \\
\hline Rest of World & $\sim 2012-2017$ & $12 \%$ & $23 \%$ & & & $15 \%$ & $26 \%$ \\
\hline \multicolumn{8}{|c|}{ 5. Chillers, air cooled (Large, $>=300$ tons $-1500 \mathrm{~kW}, 429$ ton) ${ }^{+}$} \\
\hline Asia & $\sim 2012-2017$ & $30 \%$ & $38 \%$ & & & $33 \%$ & $41 \%$ \\
\hline North America & $\sim 2012-2017$ & $23 \%$ & $32 \%$ & & & $26 \%$ & $35 \%$ \\
\hline Europe & $\sim 2012-2017$ & $35 \%$ & $42 \%$ & & & $37 \%$ & $44 \%$ \\
\hline Rest of World & $\sim 2012-2017$ & $13 \%$ & $23 \%$ & & & $17 \%$ & $26 \%$ \\
\hline
\end{tabular}




\begin{tabular}{|c|c|c|c|c|c|c|c|}
\hline \multirow[t]{3}{*}{ Countries } & \multirow[t]{3}{*}{ Year } & \multicolumn{6}{|c|}{ EE due to systems improvements and low-GWP refrigerant (\%) } \\
\hline & & \multicolumn{2}{|c|}{ HCFC-22/HFC-410A } & \multicolumn{2}{|c|}{ HC-290 } & \multicolumn{2}{|c|}{ HFC-32/ HFOs } \\
\hline & & Economic & Technical & Economic & Technical & Economic & Technical \\
\hline \multicolumn{8}{|c|}{ 1. Chillers, water cooled (Small, $<300$ tons $-500 \mathrm{~kW}, 143$ tons) ${ }^{+}$} \\
\hline Asia & $\sim 2012-2017$ & $31 \%$ & $51 \%$ & & & $34 \%$ & $53 \%$ \\
\hline North America & $\sim 2012-2017$ & $18 \%$ & $41 \%$ & & & $21 \%$ & $44 \%$ \\
\hline Europe & $\sim 2012-2017$ & $50 \%$ & $64 \%$ & & & $52 \%$ & $66 \%$ \\
\hline Rest of World & $\sim 2012-2017$ & $25 \%$ & $46 \%$ & & & $28 \%$ & $49 \%$ \\
\hline \multicolumn{8}{|c|}{ 2. Chillers, water cooled (Large, $>=300$ tons $-1500 \mathrm{~kW}, 429$ ton) ${ }^{+}$} \\
\hline Asia & $\sim 2012-2017$ & $36 \%$ & $57 \%$ & & & $39 \%$ & $59 \%$ \\
\hline North America & $\sim 2012-2017$ & $13 \%$ & $41 \%$ & & & $17 \%$ & $44 \%$ \\
\hline Europe & $\sim 2012-2017$ & $45 \%$ & $63 \%$ & & & $48 \%$ & $65 \%$ \\
\hline Rest of World & $\sim 2012-2017$ & $26 \%$ & $50 \%$ & & & $29 \%$ & $52 \%$ \\
\hline \multicolumn{8}{|c|}{ 3. Remote display cabinet - Chilled, multi-deck $(\mathrm{RVC2})^{++}$} \\
\hline Asia & 2014 & $69 \%$ & $75 \%$ & $68 \%$ & $74 \%$ & & \\
\hline North America & 2014 & $53 \%$ & $63 \%$ & $51 \%$ & $61 \%$ & & \\
\hline Europe & 2014 & $13 \%$ & $30 \%$ & $8 \%$ & $26 \%$ & & \\
\hline Rest of World & 2014 & $35 \%$ & $48 \%$ & $32 \%$ & $45 \%$ & & \\
\hline \multicolumn{8}{|c|}{ 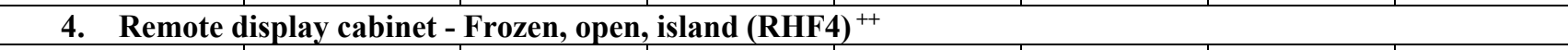 } \\
\hline Asia & 2014 & $23 \%$ & $23 \%$ & $19 \%$ & $19 \%$ & & \\
\hline North America & 2014 & $31 \%$ & $31 \%$ & $27 \%$ & $27 \%$ & & \\
\hline Europe & 2014 & $17 \%$ & $17 \%$ & $13 \%$ & $13 \%$ & & \\
\hline Rest of World & 2014 & $42 \%$ & $42 \%$ & $39 \%$ & $39 \%$ & & \\
\hline \multicolumn{8}{|c|}{ Integral display cabinet - Chilled, multi-deck $\left(\right.$ IVC2) ${ }^{++}$} \\
\hline Asia & 2014 & $66 \%$ & $77 \%$ & $64 \%$ & $76 \%$ & & \\
\hline North America & 2014 & $48 \%$ & $65 \%$ & $45 \%$ & $64 \%$ & & \\
\hline Europe & 2014 & $30 \%$ & $53 \%$ & $26 \%$ & $51 \%$ & & \\
\hline Rest of World & 2014 & $30 \%$ & $54 \%$ & $27 \%$ & $51 \%$ & & \\
\hline \multicolumn{8}{|c|}{ 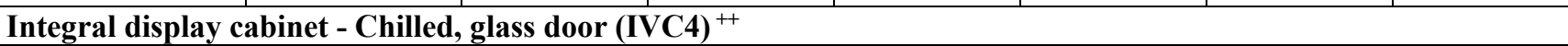 } \\
\hline Asia & 2014 & $15 \%$ & $30 \%$ & $11 \%$ & $26 \%$ & & \\
\hline North America & 2014 & $15 \%$ & $30 \%$ & $11 \%$ & $26 \%$ & & \\
\hline Europe & 2014 & $15 \%$ & $30 \%$ & $11 \%$ & $26 \%$ & & \\
\hline Rest of World & 2014 & $15 \%$ & $30 \%$ & $11 \%$ & $26 \%$ & & \\
\hline \multicolumn{8}{|c|}{ 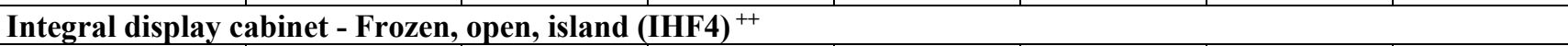 } \\
\hline Asia & 2014 & $41 \%$ & $41 \%$ & $37 \%$ & $38 \%$ & & \\
\hline North America & 2014 & $47 \%$ & $47 \%$ & $44 \%$ & $45 \%$ & & \\
\hline Europe & 2014 & $24 \%$ & $25 \%$ & $20 \%$ & $21 \%$ & & \\
\hline Rest of World & 2014 & $56 \%$ & $56 \%$ & $53 \%$ & $54 \%$ & & \\
\hline
\end{tabular}




\begin{tabular}{|c|c|c|c|c|c|c|c|}
\hline \multirow[t]{3}{*}{ Countries } & \multirow[t]{3}{*}{ Year } & \multicolumn{6}{|c|}{ EE due to systems improvements and low-GWP refrigerant (\%) } \\
\hline & & \multicolumn{2}{|c|}{ HCFC-22/HFC-410A } & \multicolumn{2}{|c|}{ HC-290 } & \multicolumn{2}{|c|}{ HFC-32/ HFOs } \\
\hline & & Economic & Technical & Economic & Technical & Economic & Technical \\
\hline \multicolumn{8}{|c|}{ Integral display cabinet - Frozen, glass lid, island $\left(\right.$ IHF6) ${ }^{++}$} \\
\hline Asia & 2014 & $32 \%$ & $34 \%$ & $29 \%$ & $31 \%$ & & \\
\hline North America & 2014 & $39 \%$ & $41 \%$ & $36 \%$ & $38 \%$ & & \\
\hline Europe & 2014 & $13 \%$ & $16 \%$ & $9 \%$ & $12 \%$ & & \\
\hline Rest of World & 2014 & $41 \%$ & $43 \%$ & $38 \%$ & $40 \%$ & & \\
\hline \multicolumn{8}{|c|}{ Domestic refrigerators/freezers (Average size) ${ }^{+++}$} \\
\hline Asia & 2015 & $16 \%$ & $60 \%$ & $22 \%$ & $63 \%$ & & \\
\hline North America & 2015 & $22 \%$ & $47 \%$ & $28 \%$ & $51 \%$ & & \\
\hline Europe & 2015 & $13 \%$ & $53 \%$ & $19 \%$ & $56 \%$ & & \\
\hline Rest of World & 2015 & $15 \%$ & $57 \%$ & $21 \%$ & $60 \%$ & & \\
\hline \multicolumn{8}{|c|}{ Beverage vending machines (500 bottle/unit capacity) ${ }^{+++}$} \\
\hline Asia & 2015 & $44 \%$ & $58 \%$ & $48 \%$ & $61 \%$ & & \\
\hline North America & 2015 & $34 \%$ & $55 \%$ & $39 \%$ & $58 \%$ & & \\
\hline Europe & 2015 & $44 \%$ & $58 \%$ & $48 \%$ & $61 \%$ & & \\
\hline Rest of World & 2015 & $19 \%$ & $59 \%$ & $25 \%$ & $62 \%$ & & \\
\hline
\end{tabular}

RID: Remote and integral displays

${ }^{+} \mathrm{HFO}$ as an alternative low-GWP refrigerant

${ }^{++} \mathrm{CO}_{2}$ as an alternative low-GWP refrigerant

${ }^{+++}$HC-600a as an alternative low-GWP refrigerant

Source: (DOE, 2011; IEA-4E, 2012a; IEA-4E, 2012b; CLASP, 2013; CLASP, 2014; IEA-4E, 2014; IEA-4E, 2015; Shah et al., 2015; Rosenquist, 2016; UNEP, 2017; DOE, 2018; DOE-FEMP, 2018) 
Table S3: Annual technical/economic electricity saving potentials in HFC reduction scenarios relative respective preKA baseline scenarios

\begin{tabular}{|c|c|c|c|c|c|c|c|c|c|}
\hline \multirow[t]{2}{*}{ Year } & \multirow[t]{2}{*}{ KA Groups } & \multicolumn{4}{|c|}{ Technical electricity savings (TWh) } & \multicolumn{4}{|c|}{ Economic electricity savings (TWh) } \\
\hline & & $\begin{array}{c}\text { SSP3 - } \\
\text { KA }\end{array}$ & $\begin{array}{c}\text { Cooling } \\
\text { for All - } \\
\text { KA }\end{array}$ & $\begin{array}{l}\text { SSP3 - } \\
\text { MTFR }\end{array}$ & $\begin{array}{c}\text { Cooling } \\
\text { for All - } \\
\text { MTFR }\end{array}$ & $\begin{array}{c}\text { SSP3 - } \\
\text { KA }\end{array}$ & $\begin{array}{c}\text { Cooling } \\
\text { for All - } \\
\text { KA }\end{array}$ & $\begin{array}{l}\text { (SSP3 - } \\
\text { MTFR) }\end{array}$ & $\begin{array}{l}\text { (Cooling } \\
\text { for All - } \\
\text { MTFR) }\end{array}$ \\
\hline \multirow[t]{5}{*}{2030} & Non-A5 (Group-I) & 806 & 806 & 767 & 767 & 491 & 491 & 455 & 455 \\
\hline & Non-A5 (Group-II) & 25 & 25 & 25 & 25 & 16 & 16 & 16 & 16 \\
\hline & A5 (Group-I) & 1452 & 1464 & 1708 & 1717 & 861 & 865 & 993 & 996 \\
\hline & A5 (Group-II) & 449 & 453 & 518 & 521 & 260 & 262 & 290 & 291 \\
\hline & Global & 2732 & 2748 & 3018 & 3030 & 1628 & 1634 & 1754 & 1759 \\
\hline \multirow[t]{5}{*}{2050} & Non-A5 (Group-I) & 1304 & 1304 & 1238 & 1238 & 795 & 795 & $\overline{734}$ & $\overline{734}$ \\
\hline & Non-A5 (Group-II) & 58 & 59 & 56 & 57 & 37 & 38 & 35 & 35 \\
\hline & A5 (Group-I) & 4017 & 4272 & 3868 & 4114 & 2474 & 2601 & 2239 & 2343 \\
\hline & A5 (Group-II) & 2503 & 2535 & 2410 & 2440 & 1515 & 1530 & 1351 & 1364 \\
\hline & Global & 7882 & 8169 & 7572 & 7849 & 4821 & 4964 & 4359 & 4477 \\
\hline \multirow[t]{5}{*}{2100} & Non-A5 (Group-I) & 1772 & 1772 & 1683 & 1683 & 1109 & 1109 & 1032 & 1032 \\
\hline & Non-A5 (Group-II) & 109 & 117 & 106 & 113 & 70 & 73 & 65 & 68 \\
\hline & A5 (Group-I) & 7341 & 9947 & 7064 & 9572 & 4345 & 5647 & 3867 & 4942 \\
\hline & A5 (Group-II) & 3581 & 3760 & 3447 & 3619 & 2100 & 2189 & 1853 & 1927 \\
\hline & Global & 12803 & 15595 & 12299 & 14987 & 7624 & 9019 & 6817 & 7969 \\
\hline
\end{tabular}




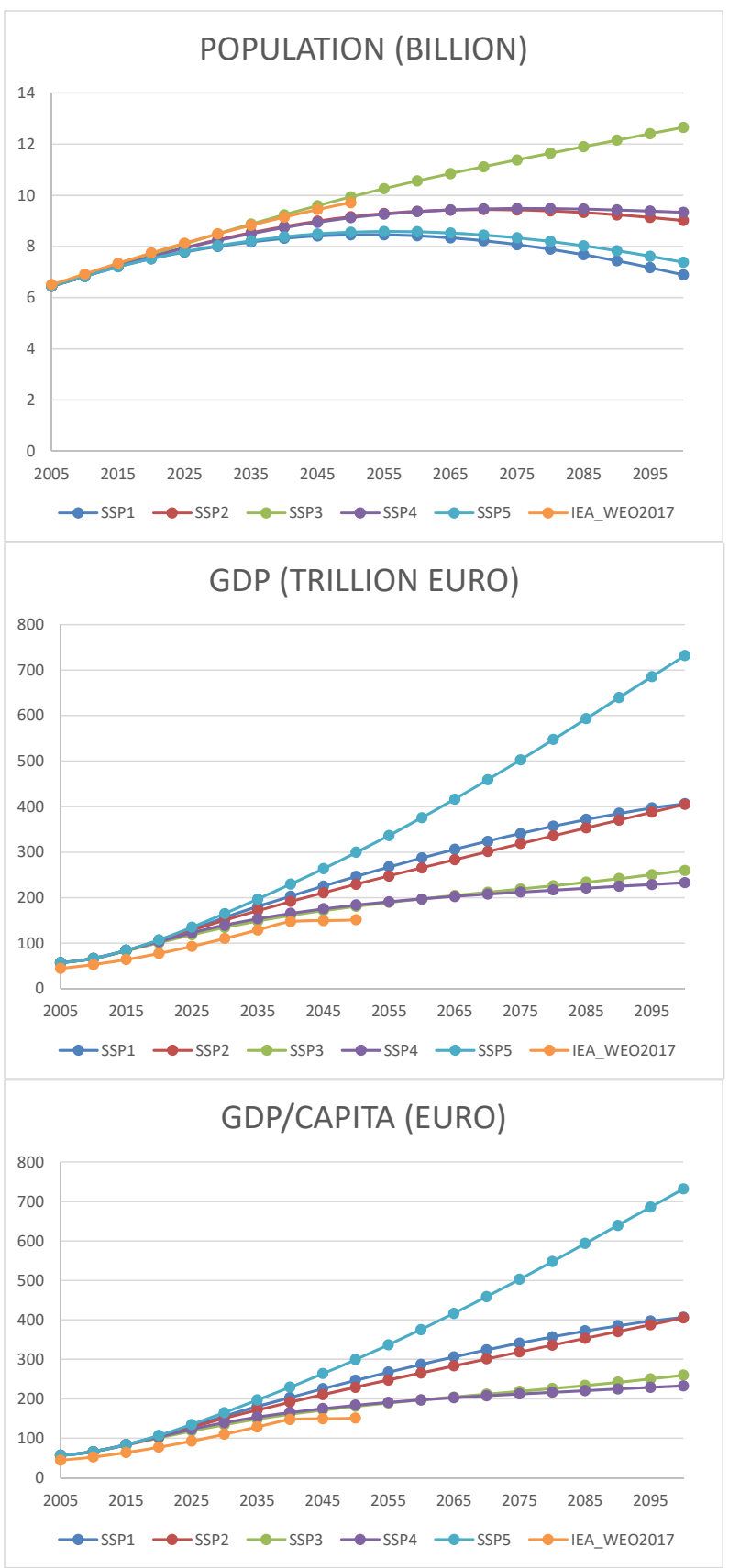

Figure S1: Comparison of future developments in global population and GDP between the IEA-WEO 2017 and the five Shared Socioeconomic Pathways (SSPs) scenarios.

Source: IIASA (2017) 

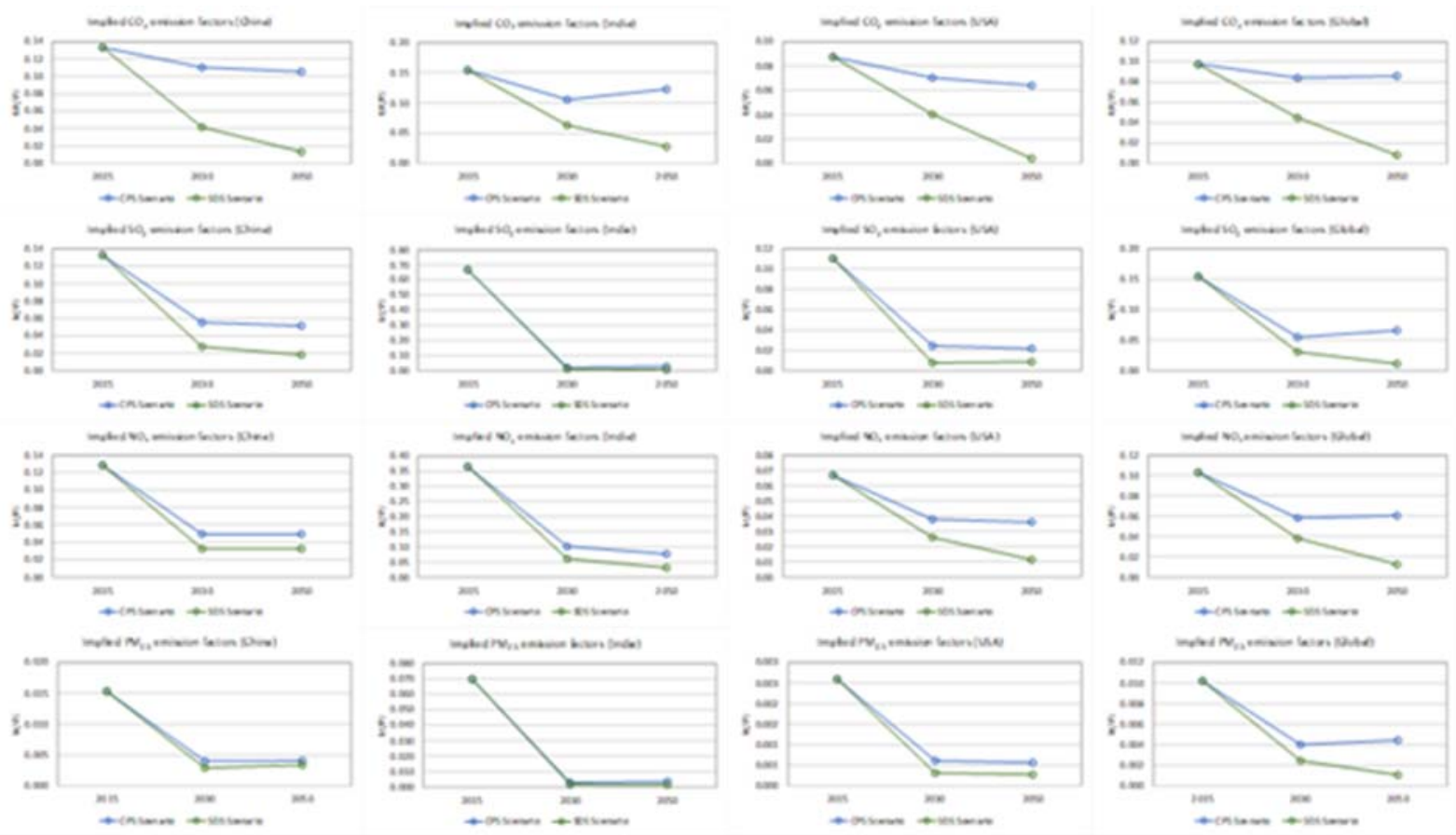

Figure S2: Implied emission factors for electricity production in the power plant sector for $\mathrm{CO}_{2}$ and the air pollutants $\left(\mathrm{SO}_{2}, \mathrm{NO}_{\mathrm{x}}\right.$ and $\left.\mathrm{PM}_{2.5}\right)$ at global and select country level

Source: IIASA/GAINS Model available at: gains.iiasa.ac.at 
a) Non-Article 5 (Group-I)

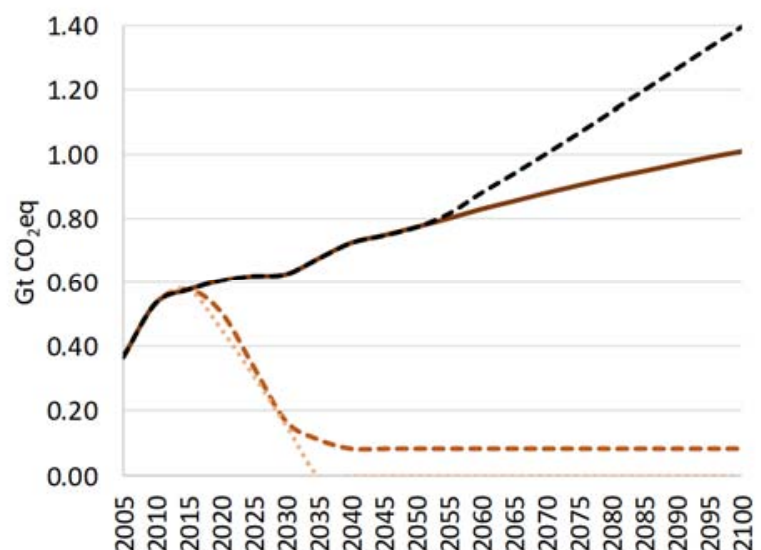

c) Article 5 (Group-I)

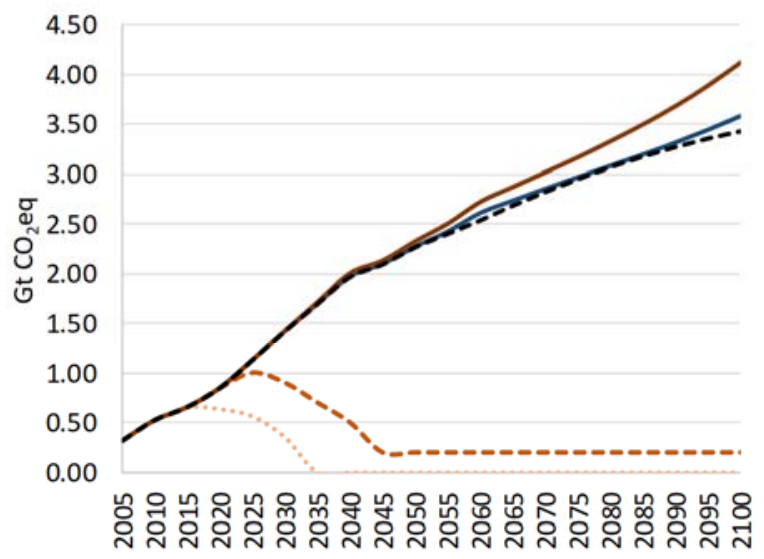

$\begin{array}{ll}\text { SSP3 -BL } & -\cdots \text { SSP3 -KA } \\ \text {---CFA -KA } & \ldots . \text { CFA -MTFR }\end{array}$ b) Non-Article 5 (Group-II)

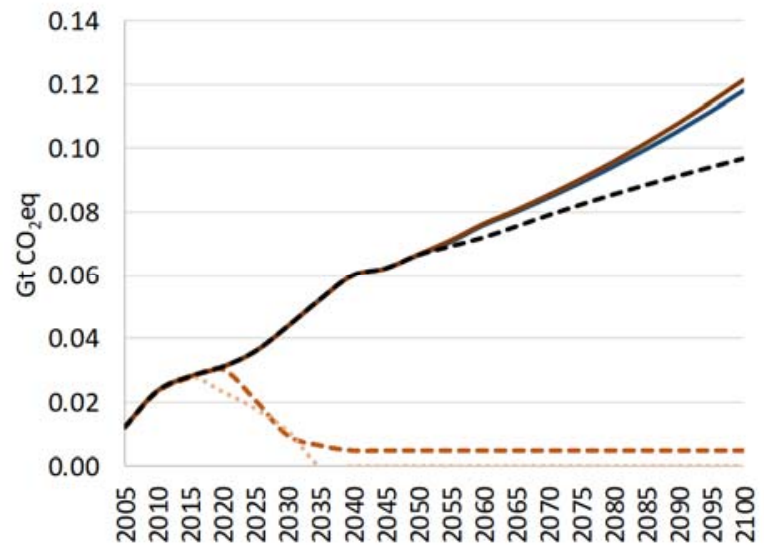

d) Article 5 (Group-I)

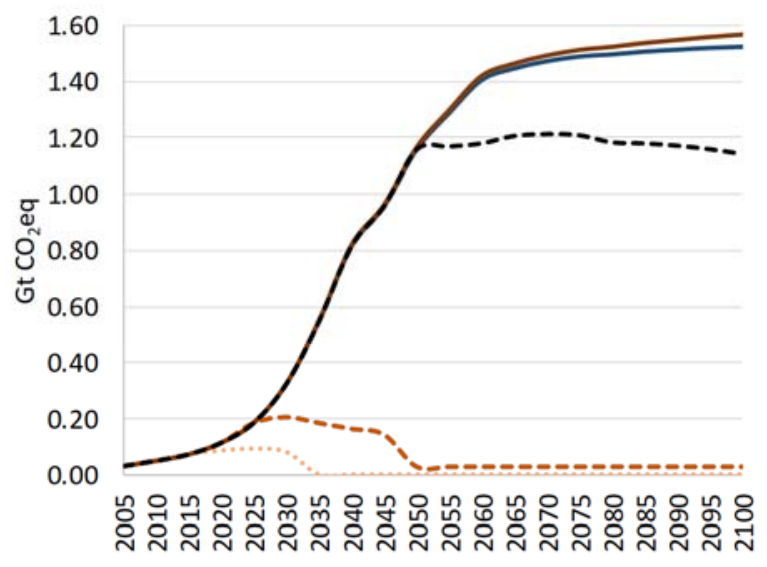

.... SSP3 -MTFR CFA -BL

----SSP1 -BL

Figure S3: Baseline HFC/HCFC (Gt CO2eq) by different party groups 
a) Non-Article 5 Group I

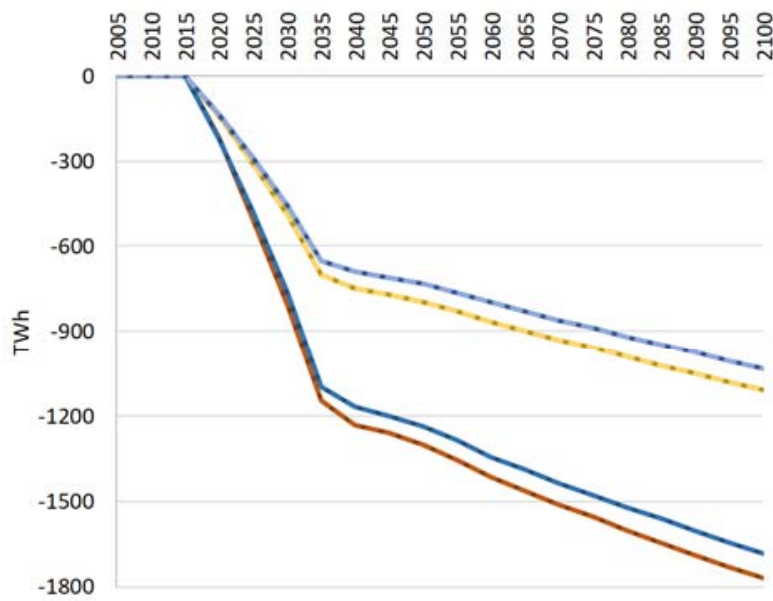

c) Article 5 Group I

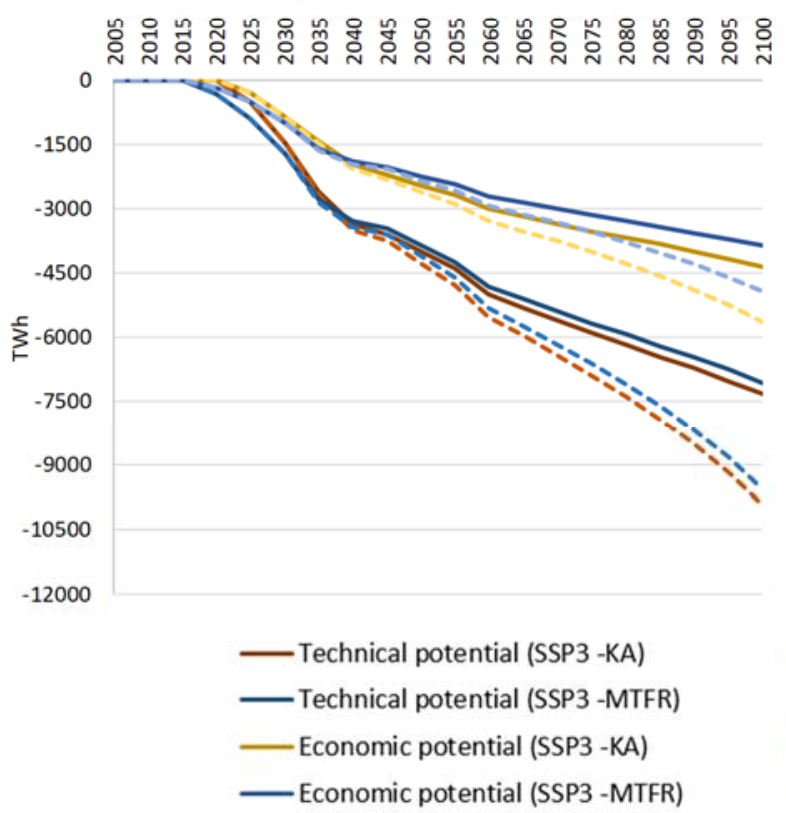

b) Non-Article 5 Group II

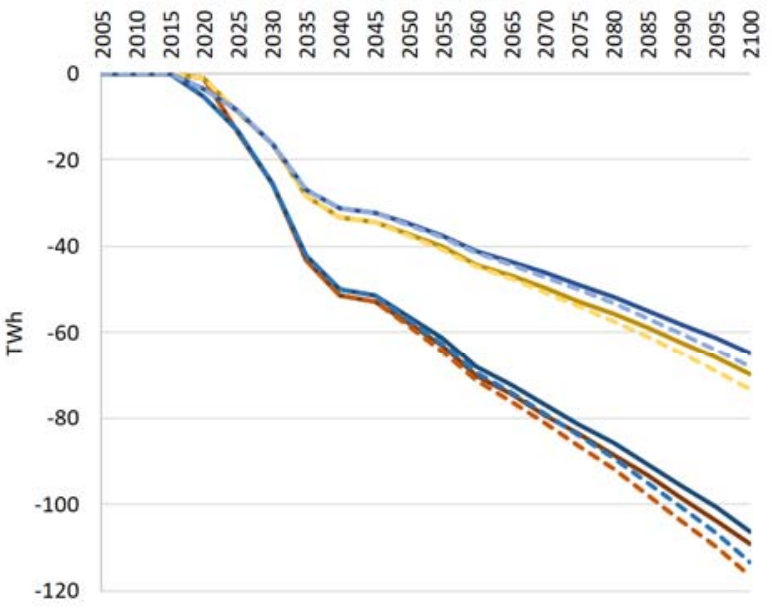

d) Article 5 Group II

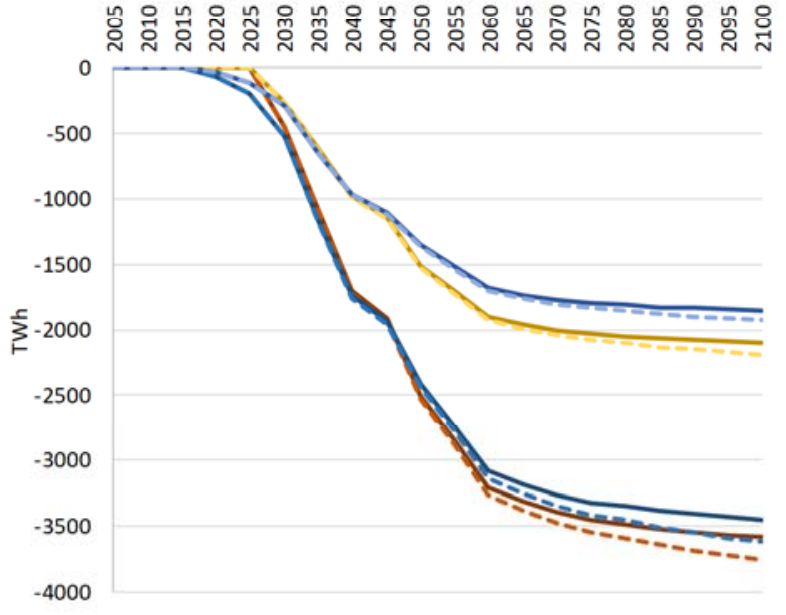

- - - Technical potential (Cooling for All -KA)

- - - Technical potential (Cooling for All -MTFR)

- - Economic potential (Cooling for All -KA)

- - Economic potential (Cooling for All -MTFR)

Figure S4: Technical and economic electricity saving (TWh) potential by different party groups 
a) Non-Article 5 Group I

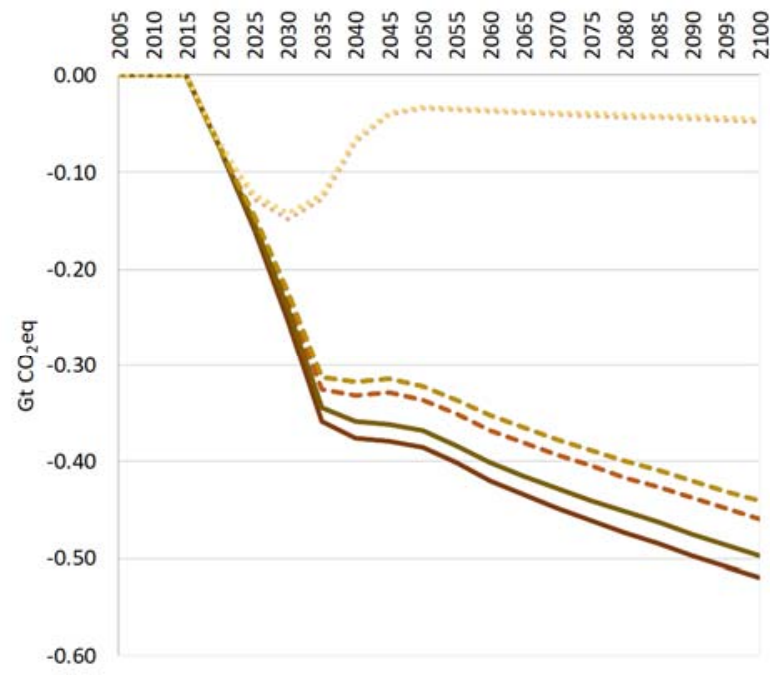

c) Article 5 Group 1

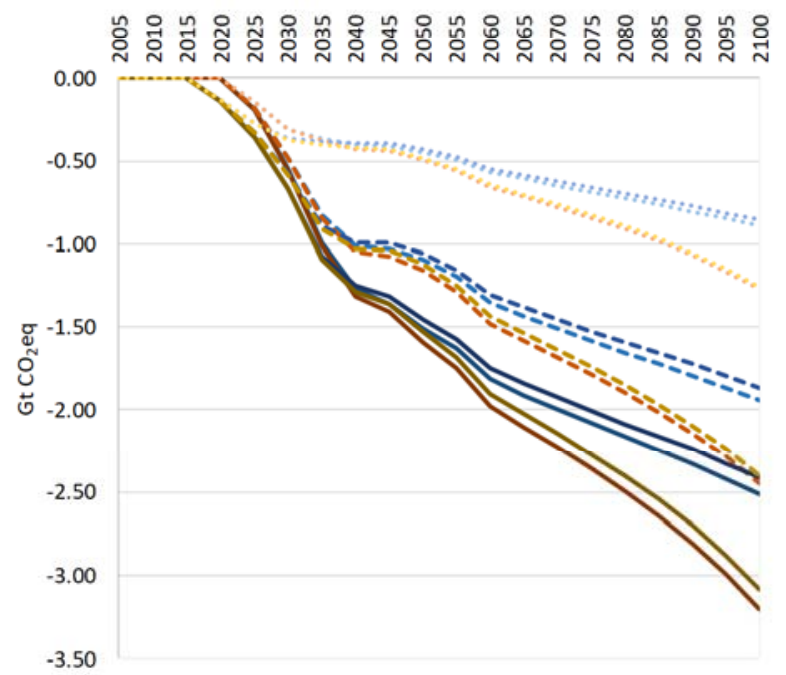

b) Non-Article 5 Group II

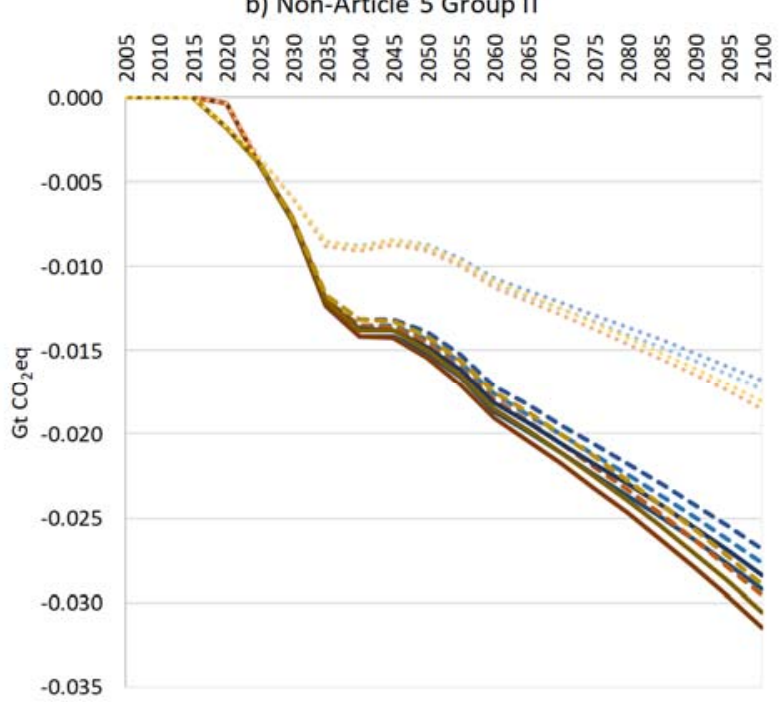

d) Article 5 Group II
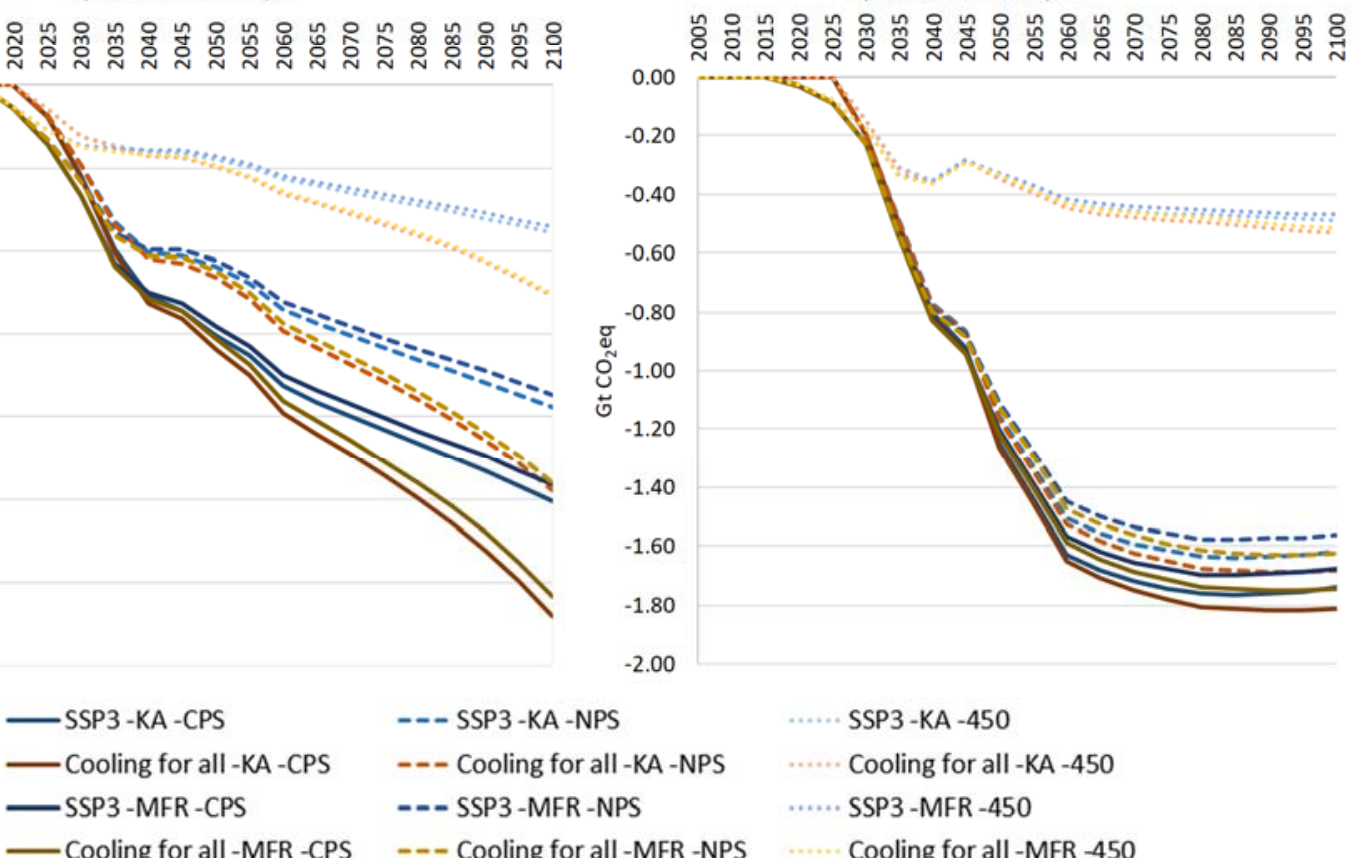

--- SSP3 -KA -NPS

--- Cooling for all -KA -NPS

--- SSP3 -MFR -NPS

--- Cooling for all -MFR -NPS

Figure S5: GHG emissions reductions in the baseline (SSP3 and Cooling for All) and alternative (KA and MTFR) scenarios due to technical electricity savings potential by different party groups 
a) Non-Article 5 Group I

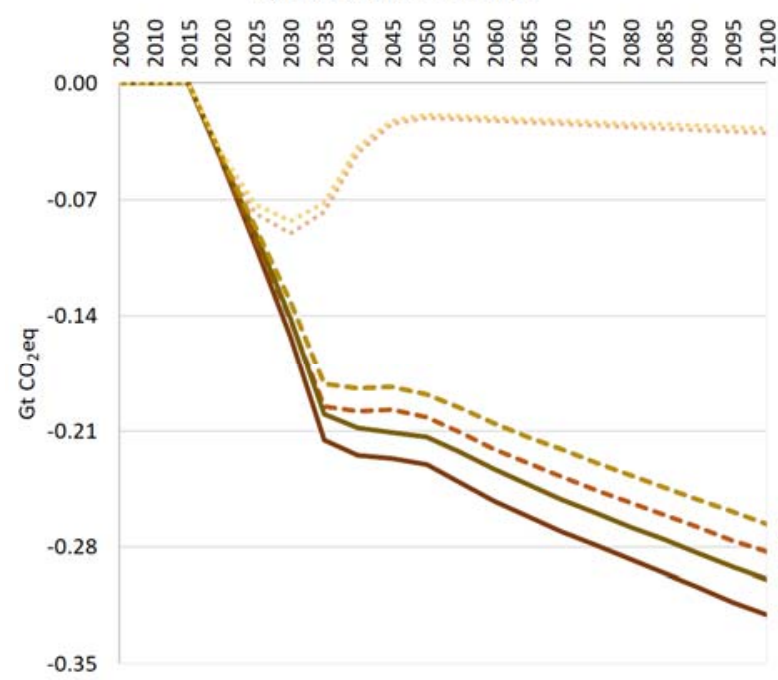

c) Article 5 Group 1

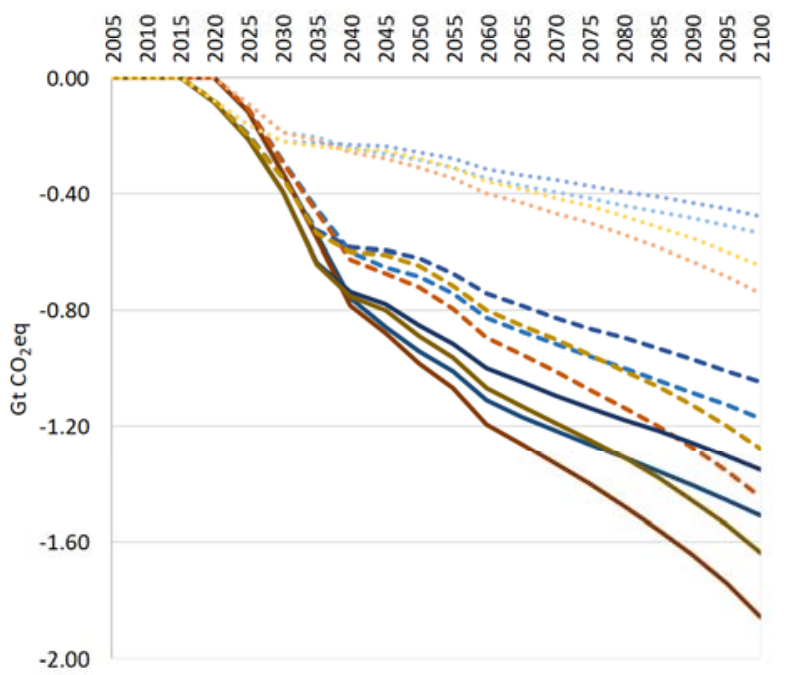

b) Non-Article 5 Group II

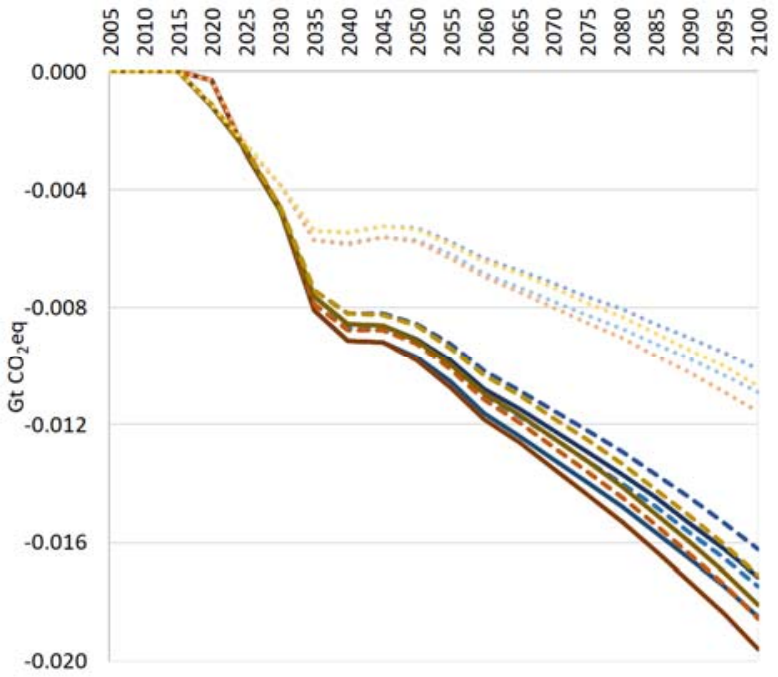

d) Article 5 Group II
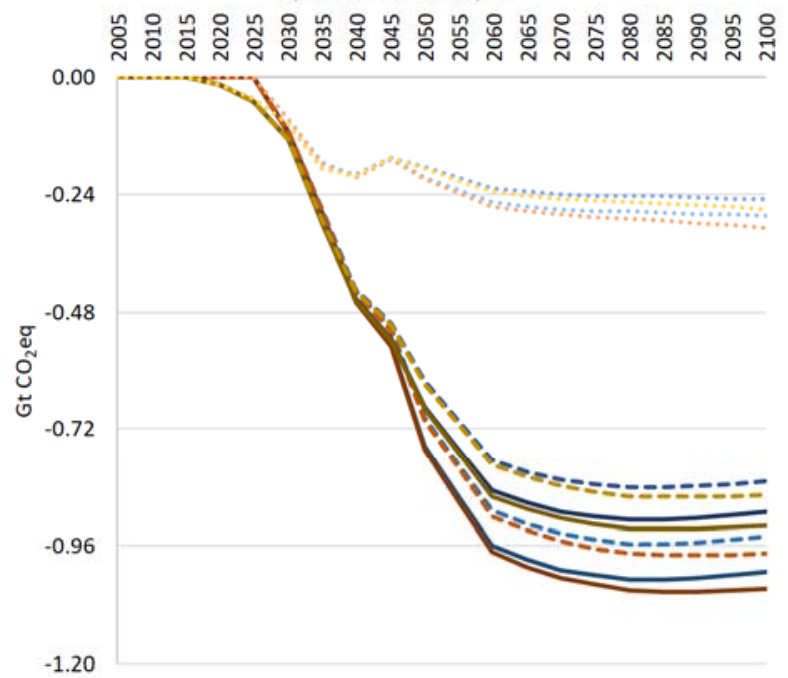

$-1.20$

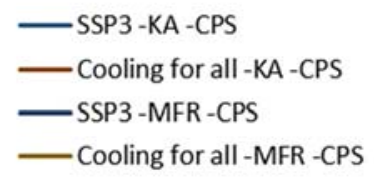

$$
\begin{aligned}
& --- \text { SSP3 -KA -NPS } \\
& --- \text { Cooling for all -KA -NPS } \\
& --- \text { SSP3 -MFR -NPS } \\
& --- \text { Cooling for all -MFR -NPS }
\end{aligned}
$$$$
\text { -..... SSP3 -KA }-450
$$$$
\text { ...... Cooling for all -KA }-450
$$$$
\text { ...... SSP3 -MFR -450 }
$$

Cooling for all -MFR -450

Figure S6: GHG emissions reductions in the baseline (SSP3 and Cooling for All) and alternative (KA and MTFR) scenarios due to economic electricity savings potential by different party groups 


\section{References}

CLASP (2013). Technical Evaluation of National and Regional Test Methods for Commercial Refrigeration Products. Collaborative Labeling and Appliance Standards Program (CLASP), Washington, D.C. (Available at: https://storage.googleapis.com/clasp-siteattachments/SEAD-Evaluation-of-National-and-Regional-TestMethods-for-Commercial-Refrigeration-Equipment.pdf accessed on 18/09/2018).

CLASP (2014). Analysis of EU policy proposals for DG ENER Lot 12 Commercial Refrigeration Including comparison with policy thresholds and product data from other regions. Collaborative Labeling and Appliance Standards Program (CLASP), Washington, D.C. (Available at: https://www.eceee.org/static/media/uploads/site-2/ecodesign/products/commercial-refrigeratorsfreezers/clasp-2014-11-analysis-eu-policy-proposals-dgenerlot12-regional-comparisons.pdf accessed on 24/09/2018).

DOE (2011). Technical Support Document for Energy Conservation Standards for Residential Refrigerators, Refrigerator-Freezers, and Freezers. Office of Energy Efficiency and Renewable Energy, U.S. Department of Energy (DOE), Washington, D.C. (Available at: https://www.regulations.gov/contentStreamer?documentId=EERE-2008-BT-STD-00120128\&content Type $=$ pdf accessed on 23/09/2018).

DOE (2018). U.S. Department of Energy's Compliance Certification Database. Washington, D.C. (Available at: https://www.regulations.doe.gov/certification-data/\#q=Product_Group_s\%3A* accessed on 12/09/2018).

DOE-/FEMP (2018). Purchasing Energy-Efficient Air-Cooled Electric Chillers. The Federal Energy Management Program (FEMP), U.S. Department of Energy (DOE), Washington, D.C. (Available at: https://www.energy.gov/eere/femp/purchasing-energy-efficient-air-cooled-electricchillers accessed on 19/09/ 2018).

EU (2006). Directive 2006/40/EC of the European parliament and of the council of 17 May 2006 relating to emissions from air-conditioning systems in motor vehicles. Official Journal of the European Union, L161: 1218.

EU (2014) Regulation (EC) No 517/2014 of the European parliament and of the council of 16 April 2014 on fluorinated greenhouse gases and repealing Regulation (EC) No 842/2006. Official Journal of the European Union, L150: 195-230.

Feng J, Yan H, Zhang B, \& Zhang J (2012). Prediction of HFC-23 Emission and Analysis of CDM Project Impact in China (in Chinese). Acta Scientiarum Naturalium Universitatis Pekinensis, 48: 310-316.

Fenhann J (2014). UNEP DTU CDM Pipeline. UNEP DTU Partnership, Copenhagen, Denmark. 
Höglund Isaksson L, Winiwarter W, \& Purohit P (2013). Non- $\mathrm{CO}_{2}$ greenhouse gas emissions, mitigation potentials and costs in the EU-28 from 2005 to 2050: GAINS model methodology. International Institute for Applied Systems Analysis (IIASA), Laxenburg, Austria.

Höglund-Isaksson L, Winiwarter W, Purohit P, Rafaj P, Schöpp W \& Klimont Z (2012). EU low carbon roadmap 2050: Potentials and costs for mitigation of non- $\mathrm{CO}_{2}$ greenhouse gas emissions. Energy Strategy Reviews, 1 (2): 97-108.

IEA-4E (2012a). Benchmarking report for refrigerated vending machines, IEA Technology Collaboration Programme on Energy Efficient End-Use Equipment (4E). International Energy Agency (IEA), Paris (Available at: https://mappingandbenchmarking.iea-4e.org/shared files/392/download accessed on 26/09/2018).

IEA-4E (2012b). Benchmarking report for Retail display cabinets, IEA Technology Collaboration Programme on Energy Efficient End-Use Equipment (4E). International Energy Agency (IEA), Paris (Available at: https://mappingandbenchmarking.iea-4e.org/shared files/453/download accessed on 26/09/2018).

IEA-4E (2014). Benchmarking report for domestic refrigerated appliances. International Energy Agency (IEA), Paris (Available at: http://mappingandbenchmarking.iea-4e.org/shared_files/526/download accessed on $\underline{22 / 11 / 2018})$.

IEA-4E (2015). Policy benchmarking for Packaged Liquid Chillers and evaluating the lack of comparability between economies. International Energy Agency (IEA), Paris (Available at: https://mappingandbenchmarking.iea-4e.org/shared files/710/download accessed on 22/11/2018).

IIASA (2017). SSP Database (Shared Socioeconomic Pathways) - Version 1.1. International Institite for Applied Systems Analysis, Laxenburg, Austria (Available at: https://secure.iiasa.ac.at/webapps/ene/SspDb/dsd?Action=htmlpage\&page=about accessed on 17/10/2018).

IPCC (2007). Climate Change 2007: The Physical Science Basis. Contribution of Working Group I to the Fourth Assessment Report of the Intergovernmental Panel on Climate Change [Solomon, S., D. Qin, M. Manning, Z. Chen, M. Marquis, K.B. Averyt, M. Tignor and H.L. Miller (eds.)], Intergovernmental Panel on Climate Change (IPCC), Cambridge University Press, Cambridge, United Kingdom and New York, USA.

METI (2015). Act on the Rational Use and Proper Management of Fluorocarbons (Act no. 64 of 2001). Ministry of Economy, Trade and Industry (METI), Tokyo, Japan.

Miller BR \& Kuijpers LJM (2011). Projecting future HFC-23 emissions. Atmospheric Chemistry and Physics, 11: 13259-13267.

Miller BR, Rigby M, Kuijpers LJM, Krummel PB, Steele LP, Leist M, Fraser PJ, McCulloch A, Harth C, Salameh P, Mühle J, Weiss RF, Prinn RG, Wang RHJ, O’Doherty S, Greally BR, \& Simmonds PG (2010). HFC-23 
(CHF3) emission trend response to HCFC-22 (CHClF2) production and recent HFC-23 emission abatement measures. Atmospheric Chemistry and Physics, 10: 7875-7890.

Montzka SA, Kuijpers L, Battle MO, Aydin M, Verhulst KR, Saltzman ES, \& Fahey DW (2010). Recent increases in global HFC-23 emissions. Geophysical Research Letters, 37: L02808.

NDRC (2015). China's Intended Nationally Determined Contribution. Submitted to the United Nations Framework Convention on Climate Change (UNFCCC), Bonn on June 30, 2015 (Available at: https://www4.unfccc.int/sites/submissions/INDC/Published Documents/China/1/China's INDC - on 2030 June 2015.pdf accessed on 12/11/2018).

Reilly, A. (2017). U.S. court rejects Obama-era plan to eliminate some potent planet warming chemicals. Science and Policy, 8 August 2017. doi:10.1126/science.aan7217.

Rosenquist, G. (2016). Energy Analysis and Environmental Impacts Division, Lawrence Berkeley National Laboratory (LBNL), Berkeley (personal communication).

Schneider LR (2011). Perverse incentives under the CDM: an evaluation of HFC-23 destruction projects, Climate Policy, 11: 851-864.

Shah N, Wei M, Letschert VE, \& Phadke AA (2015). Benefits of Leapfrogging to Superefficiency and Low Global Warming Potential Refrigerants in Room Air Conditioning. LBNL-1003671, Lawrence Berkeley National Laboratory (LBNL), Berkley, CA.

UNEI (2017). Accelerating the Global Adoption of Climate-Friendly and Energy-Efficient Refrigerators. United Nations Environment Programme (UNEP), 2017, Paris.

UNEP (2014). Ozone Secretariat - Data Access Centre. United Nations Environment Programme (UNEP), Nairobi (Available at: http://ozone.unep.org/en/data-reporting/data-centre accessed on 05/11/2014).

UNEP (2016). Further Amendment of the Montreal Protocol: Submitted by the Contact group on HFCs. TwentyEighth Meeting of the Parties to the Montreal Protocol on Substances that Deplete the Ozone Layer, United Nations Environment Programme (UNEP), UNEP/OzL.Pro.28/CRP/10, Kigali, 10-14 October 2016.

US EPA (2015). Protection of Stratospheric Ozone: Change of Listing Status for Certain Substitutes under the Significant New Alternatives Policy Program. EPA-HQ-OAR-2014-0198, U. S. Environmental Protection Agency (EPA), Washington, D.C.

Velders GJM, Fahey DW, Daniel JS, Andersen SO, \& McFarland M (2015). Future atmospheric abundances and climate forcing from scenarios of global and regional hydrofluorocarbon (HFC) emissions. Atmospheric Environment, 123: 200-209.

Wara M (2007). Is the global carbon market working? Nature, 445: 595-596. 